EPJ Web of Conferences 66, 03092 (2014)

DOI: $10.1051 /$ epjconf/ 20146603092

(C) Owned by the authors, published by EDP Sciences, 2014

\title{
Dynamics of collinear ternary fission in the fragmentation of ${ }^{252} \mathbf{C f}$
}

\author{
W. von Oertzen ${ }^{1}$, K.R. Vijayaraghavan ${ }^{2}$, and M. Balasubramaniam ${ }^{2, a}$ \\ ${ }^{1}$ Helmholtz-Zentrum Berlin, Hahn-Meitner Platz 1, 14109 Berlin, Germany \\ ${ }^{2}$ Department of Physics, Bharathiar University, Coimbatore - 641046, India
}

\begin{abstract}
With the observation of the collinear cluster tripartition in ${ }^{252} \mathrm{Cf}$, we discuss various features of the true ternary fission (collinear fission). The conditions for collinear ternary decay with three clusters are explained. We discuss the potential energy surfaces for ternary decay and conditions for the collinear decay.
\end{abstract}

\section{Collinear ternary fission}

Ternary fission with comparable masses is a process which occurs in heavy nuclei under conditions of large values of $\mathrm{Z}^{2} / \mathrm{A}$, called the fission-parameter. The decay into three fragments (true ternary fission) observed is found to be collinear. Recent experiments for the spontaneous decay of ${ }^{252} \mathrm{Cf}(\mathrm{fff})$ and of neutron induced fission in ${ }^{235} \mathrm{U}(\mathrm{n}$,fff), using the missing mass approach with two FOBOS-detectors in coincidence at $180^{\circ}$, see ref. [1,2], true ternary fission has been observed with the missing mass method. In this new kind of radioactive fission decay, the so called collinear cluster tripartition (CCT), the three fragments are emitted collinear, here typical fragments are isotopes of $\mathrm{Sn}, \mathrm{Ni}$, and $\mathrm{Ca}$. The latter, $\mathrm{Ca}$, as the third particle is positioned along the line connecting $\mathrm{Sn}$ and Ni. This exotic decay can be understood as a breakup of very elongated (hyper-deformed) shapes with two neck ruptures.

From three different experiments reported in ref. [1,2] on CCT, an overall yield of $4 \times 10^{-3} /$ (binary fission) has been observed. This result has been considered to have unusually high values, when compared with the previously known yields in "ternary fission" with the emission of lighter fragments perpendicular to the fission axis [3, 4].

This high probability can be explained if the phase space for the CCT-decay is considered (see also $[5,6]$ ). The phase space depends on, i)the potential energy surfaces (PES), ii) the Q-values, the latter determining the number of possible isotope combinations, iii) the excitation energy range in the individual fragments, iv) the momentum range, $v$ ) the number of excited states (density of states) in combinations consisting of 2(3) isotopes, vi) the number of excited states in each fragment, and vii) the spin (J) multiplicity in these states with spins up to $(6-8)^{+}$(phase space factor $(2 \mathrm{~J}+1)$ ). Fragments with odd numbers of protons and/or neutrons have in addition a particular large density of states.

The question of the relative importance of fission through oblate or prolate shapes (chain configurations) has been addressed repeatedly [7-9]. Within the three cluster model, it has been shown, that the collinear configuration is the favoured mode over the oblate configuration [9] for the ternary

ae-mail: m.balou@gmail.com

This is an Open Access article distributed under the terms of the Creative Commons Attribution License 2.0, which permits unrestricted use, distribution, and reproduction in any medium, provided the original work is properly cited. 


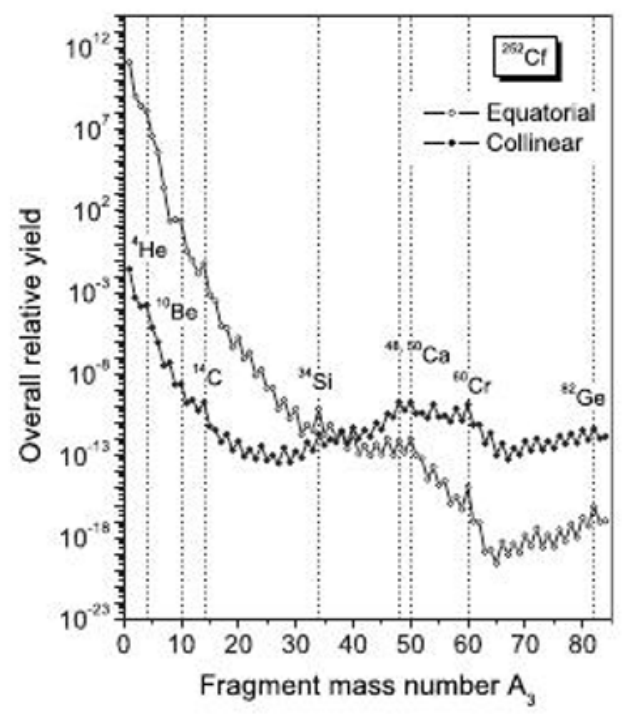

Figure 1. Curves for relative yields in ternary decays of ${ }^{252} \mathrm{Cf}$ (sf) are compared (from ref. [9]) for oblate (equatorial, triangular) and prolate (collinear) geometries. Prolate fission dominates for heavier third fragments.

decays (see Fig.1). In this figure the relative yields of true ternary fission is shown as function of the mass of the third fragment. For masses (charges) heavier than $\mathrm{A}=35-40$ the prolate fission starts to dominate. This fact is due to the dominance of the Coulomb interaction, the central fragment gives a shielding effect to the fragments at the outer borders. In addition the Coulomb interaction between the fragments at the borders is minimized.

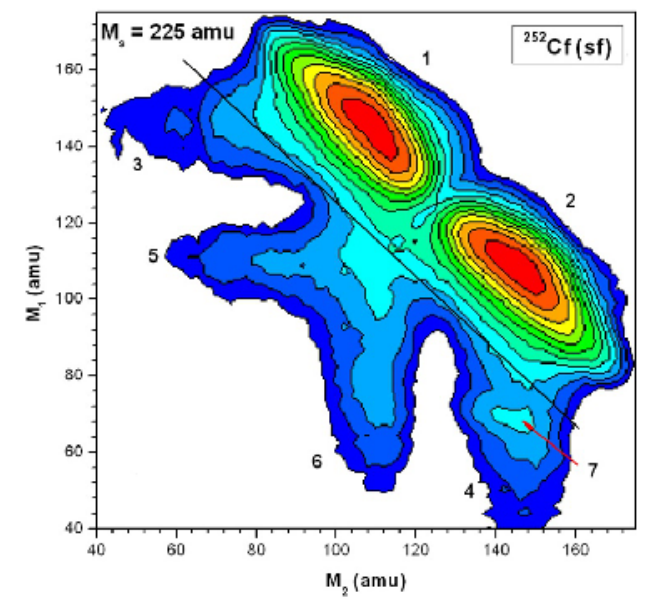

Figure 2. (Color online) The coincidence matrix for binary coincidences of Masses $\mathrm{M}_{1}$ and $\mathrm{M}_{2}$ as observed in refs [1,2]. A line with the sum of the masses $M_{S}=225$ is shown for illustration. The position of the missing mass peak is labelled as 7 . The missing mass $\mathrm{M}_{3}$ are isotopes of $\mathrm{Ca}$, see refs. $[1,2]$ for details.

In CTT-decay we have favorable dynamical conditions for the sum of fragments with mass combinations: ${ }^{60-70} \mathrm{Ni}$ with ${ }^{130-134} \mathrm{Sn}$, and ${ }^{50-58} \mathrm{Ca}$. With the large Q-values of the mass partitions in CCT events, the total phase space (discussed also in ref. [5, 10]) of the CCT-decay is factors of ca. 300010000 larger if compared with the phase space in the ternary decays [3] with one particular mass combination, and one light particle, the processes usually named "ternary fission". 
(a)

(b)

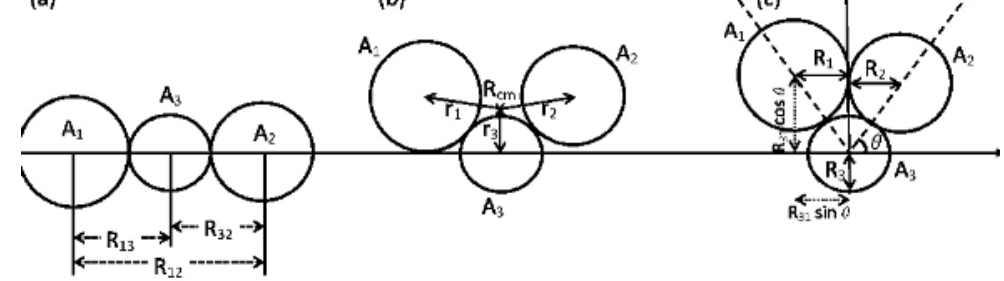

Figure 3. (Color online) Geometrical illustration of the position of three fragments with deviations from a collinear configuration, defined by an angle $\theta$. The radius vector $R_{x}$ of the fragments and the component vectors between the fragments 1 and 3 are labelled.

Further, a study of the kinematics [5] for the collinear breakup into isotopes of $\mathrm{Sn}+\mathrm{Ca}+\mathrm{Ni}$ indicates, that very low kinetic energies of the third particle occur (positioned in between the other two fission fragments). Because of this fact, this mode may sofar have escaped the experimental detection.

The previously studied ternary decays $[3,4]$ with lighter fragments, perpendicular to the fission axis from an oblate configuration give decreasing yields as function of the mass(charge) of the third particle [4]. The yields of fragments $A_{3}$ in the region of mass $=40$ became very small. From this work and in a previous survey of the theoretical predictions of the last decades ([10]) it became clear, that for larger charge (mass) of the third fragment, $A 3$, the ternary decay will be collinear. The collinear aligned multi-cluster configurations are energetically the optimum configurations for multi cluster decays. From potential energy (PE) calculations and from the PE shown in Fig.4 we conclude, that the arrangement with $\mathrm{Ca}$ as a third fragment in the center gives a lower value of the potential energy and that this arragement is favored in agreement with the experiment (Fig.1). In Fig.4 we further illustrate the reason for $\mathrm{Ca}$-isotopes as the missing mass, the arrangement with $\mathrm{Ni}$ at the center has a $\mathrm{PE}$, which is less favorable by $10 \mathrm{MeV}$.

The name "ternary" fission has been used sofar for decays, when a third light particle is emitted perpendicular (or at some finite angle) to the binary fission axis [3, 4]. All experimental observations and numerous theoretical predictions suggest, that the collinear configuration is preferred relative to the oblate configuration for heavy ternary fragments.

In a previous publication [5] we had studied the kinematics of the collinear fission decay. We have used for this study the assumption of a sequential decay corresponding to two neck ruptures. These neck configurations are connected to the concept of hyper-deformation. The time sequence of the two random (in time) ruptures is connected to the fission times. In the experimental observations it is assumed (see ref. [5]) that after a first rupture into two fragments $\mathrm{A} \rightarrow \mathrm{A} 1+\mathrm{A} 23$, the second fragment $\mathrm{A} 23$ fissions into the final nuclei $\mathrm{A} 23 \rightarrow \mathrm{A} 2+\mathrm{A} 3$. The initial fission axis is preserved and the two fragments $A 2$ and $A 3$ are emitted into the same direction, and one of them in the opposite direction of fragment $A 1$, they remain collinear (relative angle 0 degrees). The collinear fragments can be separated by a specific experimental effect. In the experiments [1], an angular dispersion is created by a foil in the direction of one of the detector arms, arm 1 , for the two fragments $A 2$ and $A 3$. The resulting angular opening of the two vectors of these two fragments allows the blocking of one of the fragments in a specific blocking structure in the detectors. In this way a missing mass event is created in a coincidence study, where the observed parameters correspond to a complete set of variables $\left(180^{\circ}\right.$ relative angle, two masses, and two energies). The result is a binary mass M3-M2 coincidence plot, where the missing mass appears with the sum of the two masses smaller then $\mathbf{M}_{S}=252$. In Fig. 2 the 


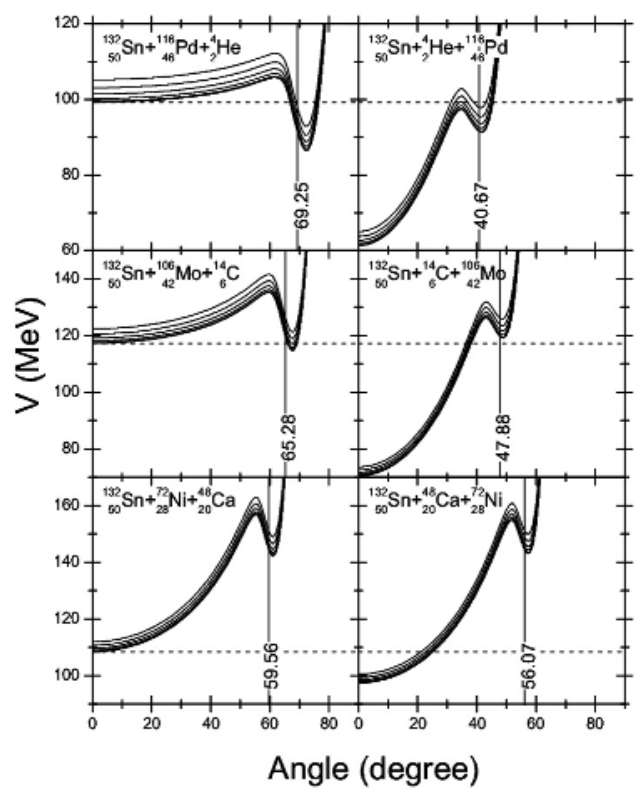

Figure 4. (Color online) The total ternary fragmentation potential as a function of angle $\theta$ is presented for three different fragment combinations. The horizontal line corresponds to triangular configurations and the value of the touching angle is labelled.

two major blobs are due to binary fission, with background due to the scattering from the blocking structure. The missing mass events are found in the local maximum labelled, 7.

In the present work [6], we discuss the competition between collinear and oblate triangular configurations in the ternary fragmentation of ${ }^{252} \mathrm{Cf}$, related to the question of collinearity. For the prescission shapes the potential energies are calculated as a function of the angle $\theta$ of the third fragment (A3) (see Fig. 3) relative to the two other fragments (A1, A2).

The potential is calculated as a function of the angle $\theta$ between the $x$ axis and the line passing through the centers of the middle fragment and the other two fragments. The potential is calculated from an angle $\theta=0$, corresponding to a collinear arrangement of the three fragments, as shown in Fig. 3 and arrangements corresponding to a triangular configuration reached for some angle (referred to as the touching angle), where all three fragments surfaces are touching each other, as shown in part (c) in Fig.3. A narrow valley is observed which forces the fragments into collinear configurations.

\section{References}

[1] Yu. V. Pyatkov et al. Eur. Phys. J. A 45, 29 (2010)

[2] Yu. V. Pyatkov et al. Eur. Phys. J. A 48, 94 (2012)

[3] F. Gönnenwein, Nucl. Phys. A 734, 213 (2004)

[4] F. Gönnenwein, EurophysicsNews 36/1, 11 (2005)

[5] K. R. Vijayaraghavan, W. von Oertzen and M. Balasubramaniam, Eur. Phys. J. A 48, 27 (2012)

[6] K. R. Vijayaraghavan, M. Balasubramaniam and W. von Oertzen, to be published

[7] D. N. Poenaru, R. A. Gherghescu, W. Greiner, Nucl. Phys. A 747, 182 (2005)

[8] H. Diehl, W. Greiner, Nucl. Phys. A 229, 29 (1974)

[9] K. Manimaran et al., Phys. Rev. C. 83, 034609 (2011)

[10] W. von Oertzen, Y.V. Pyatkov and D. Kamanin, Acta Phys. Polonica 44, 447 (2013)

[11] G. Royer, J. Phys. G: Nucl. Part. Phys. 21, 249 (1995) 